\title{
Editorial
}

\section{HIV Epidemic Situation in Bangladesh: An Overview}

\author{
Received: February 9, 2017 Accepted: March 29, 2017 \\ doi: http://dx.doi.org/10.3329/jemc.v7i2.32649
}

Bangladesh is one of the countries of the world where there is low human immunodeficiency virus (HIV) prevalence, but is still considered to be at high risk because of presence of many risk factors for spread of HIV. The national prevalence among the key population groups remains low - people who inject drug (PWID) $(1.1 \%)$, female sex worker (FSW) $(0.3 \%)$, male sex worker (MSW) (0.4\%), men who have sex with men (MSM) $(0.4 \%)$ and transgender (TG)/hijra (1.0\%); but it remains extremely vulnerable due to its socioeconomic and cultural settings. ${ }^{1}$ After all, knowledge and practice are reducing risk factors of getting HIV in Bangladesh and higher level of socio-demographic and economic status, especially among the men work to prevent HIV. ${ }^{2}$ The first case of HIV in Bangladesh was detected in 1989 and till December 2015 the total number of detected cases was 4143 , of whom 658 died, leaving 3485 known people living with HIV. Less than one-third of detected people living with HIV are women; but the majority of infections are likely to remain undetected, and the total national estimate is about 9,000 people living with HIV. HIV prevalence has never exceeded $0.1 \%$ in the general population and has remained below $1 \%$ for most key populations. $^{3}$

According to the NASP (2016) report, key populations have high HIV prevalence in certain geographical areas. Among the TG/Hijras, the HIV prevalence was $7.1 \%$ in Hilli which is a small border town in the Northwestern part of Bangladesh bordering the Indian State of West Bengal. ${ }^{4}$ The prevalence was $1.6 \%$ among casual sex workers in Hilli though HIV prevalence was below $1 \%$ in most groups of female sex workers; on the other hand, HIV prevalence among males who inject drugs in Dhaka city has increased steadily over the years from $1.4 \%$ in 2000 to $7.0 \%$ in 2007 and it was $5.3 \%$ in $2011 .{ }^{4}$ The pattern of behaviors that boost the spread of the HIV infection is well established in the Bangladesh society. ${ }^{5,6}$ By the study of icddr,b (2011) among male injecting drug users indicated that nearly $44.2 \%$ of the PWID share needles and syringes and PWID male visiting female sex workers was estimated $21.9 \% .^{7}$ Although a significant portion of the male injecting drug users reported to use condom during their last sex with sex workers, condom use with their regular partners or spouses was reported by only $27.7 \%{ }^{7}$

Selling sex to procure drugs is quite common in many parts of Bangladesh. Evidence shows that some female drug users in Bangladesh turn to sex workers out of financial necessity to support their addiction. ${ }^{8}$ The overlap between sex work and injecting drug use is considered as one of the most dangerous conditions for rapid spread of HIV and other sexually transmitted infections (STIs) and there are more possibilities for transmission to the general population. ${ }^{9}$ Women who are involved in commercial sex are very often largely dependent on their partners for the procurement and use of drugs. There are well-documented reports of risk behaviors among MSM in Bangladesh. A behavioral survey conducted in a sample of MSM in Dhaka indicated that $71 \%$ of MSM reported having anal sex with commercial or non-commercial male partners within the month prior to the survey, and only $26 \%$ reported use of condoms during their last anal sex. Moreover, $30 \%$ of the respondents bought sex from female sex workers and another $46.7 \%$ purchased sex services from male sex workers in the last one month preceding the survey. It is also found that nearly $17.7 \%$ of MSM reported at least one symptom of sexually transmitted infection (STI) in the past one year. ${ }^{10}$

A recent behavioral survey conducted by icddr, $b$ in 2010 among TG/Hijras in Dhaka reported that most TG/Hijra (87.5\%) had anal sex with male partners in the last month and only $19.4 \%$ used condom during the last sex act. ${ }^{11}$ A substantial proportion of youth have multiple sex partners; drug users share and reuse their needles; sex workers have poor condom use and high STI prevalence; unscreened blood transfusion and increasing high-risk sexual behaviors are common. ${ }^{6}$ The frequency of pre-marital and extramarital sex and the large number of sexual acts with 
sex workers are commonly known factors associated with the transmission of HIV in Bangladesh. Among the general population, approximately $10 \%$ of men reported purchase of sex from female sex workers. ${ }^{12}$ Another study on unmarried young people revealed that almost one in three ( 28 percent) of them reported one or more symptoms of STI in the past 12 months. ${ }^{6}$ All these risk behaviors have been contributing to the continued HIV transmission among key population groups and, if appropriate measures are not taken, it may spread to the general population. To overcome the situation, appropriate intervention should increase comprehensive knowledge on HIV and STIs across communities and also the information on sexual and reproductive health as well as importance of safer sexual practices, condom usage, use of sterile injecting equipment and access to treatment for STIs needs to be disseminated among both the communities and the key population. It is also recommended to ensure age disaggregated monitoring of prevention, care and treatment programs in all 64 districts of Bangladesh.

\section{Akkur Chandra Das}

M Phil student, Department of Population Sciences

University of Dhaka, Bangladesh and

Program Officer, BCCM Secretariat,

Ministry of Health and Family Welfare

Government of the People's Republic of Bangladesh

Email: akkurdas@gmail.com

\section{References}

1. National AIDS/STD programme (NASP). Investment case: prioritizing investment options in HIV response in Bangladesh to end AIDS by 2030, with financial and technical assistance from UNAIDS, 2015.

2. Sultana R, Das AC. Knowledge and practice regarding HIV among urban-rural men in Bangladesh: a cross sectional study. Bangladesh J Infect Dis 2015; 2(1): 3-8.

3. National AIDS/STD programme (NASP). Assessment of impact of harm reduction interventions among people who inject drugs (PWID) in Dhaka city. Dhaka,
Bangladesh: Ministry of Health and Family Welfare, Directorate General of Health Services, December, 2014.

4. National AIDS/STD programme (NASP). Mapping study and size estimation of key populations in Bangladesh for HIV programs 2015-2016. June 2016: $1-191$.

5. Ministry of Health and Family Welfare (MOHFW)/ Save the Children and ICDDR,B. Endline HIV/AIDS survey among youth in Bangladesh. Dhaka: MOHFW, Directorate General of Health Services, National AIDS/STD Programme (NASP), 2008.

6. Ministry of Health and Family Welfare (MOHFW). Baseline HIV/AIDS survey among youth in Bangladesh - 2005 "Project on prevention of HIV/ AIDS among young people in Bangladesh." National AIDS/STD Programme (NASP), Directorate General of Health Services, 2005.

7. International Centre for Diarrhoeal Disease Research, Bangladesh (icddr,b). Assessing the effectiveness and quality of harm reduction services for injecting drug users delivered through their peers in Dhaka and identifying gaps and barriers to effective service provision. Dhaka: icddr,b; report submitted to Save the Children, USA, December 2011.

8. Azim T, Chowdhury EI, Reza M, Ahmed M, Uddin MT, Khan R et al. Vulnerability to HIV infection among sex worker and non-sex worker female injecting drug users in Dhaka, Bangladesh: evidence from the baseline survey of a cohort study. Harm Reduction Journal 2006; 17(3): 33.

9. UNAIDS/World Bank. 20 Years of HIV in Bangladesh: experiences and way forward. Dhaka: UNAIDS, 2009.

10. International Centre for Diarrhoeal Disease Research, Bangladesh (icddr,b). A behavioral survey of MSM in Dhaka. Dhaka: icddr,b, 2010.

11. International Centre for Diarrhoeal Disease Research, Bangladesh (icddr,b). A behavioral survey of hijra in Dhaka. Dhaka: icddr,b, 2010.

12. Family Health International/icddr,b. Male reproductive health survey. Dhaka: FHI/icddr,b, 2006. 